\title{
Low Temperature Thermodynamics of Spin-1/2 XX Chains with Periodically Embedded Impurities
}

\author{
E. EzERSKAYA* AND V. ChERANOVSKiI \\ V.N. Karazin Kharkiv National University, Svoboda sqr. 4, 61022 Kharkiv, Ukraine
}

\begin{abstract}
Using standard transfer-matrix scheme, we performed numerical simulation of the field and the temperature dependences of the magnetization and the heat capacity for exactly solvable quantum model based on spin- $1 / 2 \mathrm{XX}$ chain with the periodically embedded Ising spins.
\end{abstract}

DOI: 10.12693/APhysPolA.137.631

PACS/topics: Heisenberg spin chain models, low-temperature magnetic properties

\section{Introduction}

One-dimensional effective spin models of nanostructured magnetic materials like polymeric complexes of transition metals are permanently attracting big interest of the investigators due to their interesting physics and potential applications in nanoelectronic devices and robust quantum communications [1-3]. On the other side, some of these models can be solved exactly. This gives very important opportunities for the verification of numerous approximate methods in quantum theory of lowdimensional magnetism [1].

We proposed exactly solvable 1D quantum spin model based on spin- $1 / 2 \mathrm{XX}$ chain with the periodically embedded equal Ising spins. This model can be treated as the spin- $1 / 2 \mathrm{XX}$ chain system decorated by the impurity Ising spins. The energy spectrum of this model has a relatively simple structure for arbitrary values of equal embedded spins. We derived the corresponding exact dispersion relations and determined the conditions for the appearance of the localized energy levels at arbitrary value of embedded Ising spins. On the basis of these results we performed some numerical studies of the lowtemperature magnetic properties of the model proposed with the help of standard transfer matrix technique.

\section{Model}

Let us consider a set of finite XX spin $1 / 2$ chains connected by Ising spins $S$ into the "ring". The corresponding Hamiltonian has the following form:

$$
\begin{aligned}
\hat{\boldsymbol{H}} & =-\sum_{l=1}^{L}\left\{g_{0} \mu_{\mathrm{B}} H \sigma_{l}^{z}+\left[\sum_{n=1}^{N} g \mu_{\mathrm{B}} H S_{l, n}^{z}\right.\right. \\
& +J_{0}\left(\sigma_{l}^{z} S_{l, 1}^{z}+\sigma_{l+1}^{z} S_{l, N}^{z}\right) \\
& \left.\left.+J \sum_{n=1}^{N-1}\left(S_{l, n}^{x} S_{l, n+1}^{x}+S_{l, n}^{y} S_{l, n+1}^{y}\right)\right]\right\} .
\end{aligned}
$$

\footnotetext{
*corresponding author; e-mail: yezerska@karazin.ua
}

Here $J>0$ is the exchange integral for spin- $1 / 2 \mathrm{XX}$ chain, $J_{0}$ is the exchange integral of the Ising (or $z z$ )-type interactions with impurity spins, $\mu_{\mathrm{B}}$ is the Bohr magneton, $g$ and $g_{0}$ are $g$-factors for chain and Ising impurities respectively, $H$ is the longitudinal uniform magnetic field, $N$ is the number of spins in each XX chain, and $L$ is the number of chains.

For the model Hamiltonian (1) z-projections of the impurity spins are the good quantum numbers, so these Hamiltonians may be treated as a set of Hamiltonians of finite spin-1/2 XX chains with the effective "impurity" spins at the ends, considering $\sigma_{l}=-S, \ldots, S$ as the parameter of the Hamiltonian. Taking into account the periodicity, one can show that the Hamiltonian (1) has the form

$$
\hat{\boldsymbol{H}}\left(\sigma_{1}, \ldots, \sigma_{L}\right)=\sum_{l=1}^{L} \hat{\boldsymbol{H}}_{l}\left(\sigma_{l}, \sigma_{l+1}\right), \quad \sigma_{L+1} \rightarrow \sigma_{1} .
$$

By means of the Jordan-Wigner transformation (see, for example [1, 2]) this Hamiltonian can be rewritten as

$$
\begin{gathered}
\hat{\boldsymbol{H}}_{l}=E_{0}\left(\sigma_{l}, \sigma_{l+1}\right)+\left(h+J_{0} \sigma_{l}\right) a_{l, 1}^{\dagger} a_{l, 1} \\
+\left(h+J_{0} \sigma_{l+1}\right) a_{l, N}^{\dagger} a_{l, N}+h \sum_{n=2}^{N-1} a_{l, n}^{\dagger} a_{l, n} \\
-\frac{J}{2} \sum_{n=1}^{N-1}\left(a_{l, n}^{\dagger} a_{l, n+1}+a_{l, n+1}^{\dagger} a_{l, n}\right) .
\end{gathered}
$$

Here $E_{0}\left(\sigma_{l}, \sigma_{l+1}\right)=-\left[\left(g_{0} \mu_{\mathrm{B}} H+J_{0}\right)\left(\sigma_{l}+\sigma_{l+1}\right)+g \mu_{\mathrm{B}} H N\right] / 2$, $h=g \mu_{\mathrm{B}} H$.

We solved one particle Schrödinger equation in the lattice site representation for (3) and derived the dispersion relations for the exact "excitation" energies

$$
\varepsilon_{\sigma_{l} \sigma_{l+1}}=h-\frac{J}{2}\left(x+\frac{1}{x}\right) .
$$

Here the parameters $x$ obey the following equation:

$$
\left(\alpha_{1} x+1\right)\left(\alpha_{2} x+1\right)-\left(\alpha_{1}+x\right)\left(\alpha_{2}+x\right) x^{2 N}=0,
$$

were we used the notations $\alpha_{1}=2 J_{0} \sigma_{l} / J$, $\alpha_{2}=2 J_{0} \sigma_{l+1} / J$. Due to the structure of above equation, there are two types of solutions $x=\exp \left(\mathrm{i} k_{\sigma_{l} \sigma_{l+1}}\right)$, which correspond to the quasi-continuous energy band 


$$
\varepsilon_{k_{\sigma_{l} \sigma_{l+1}}}=h-J \cos k_{\sigma_{l} \sigma_{l+1}},
$$

and real valued $\left|x_{\sigma_{l} \sigma_{l+1}}\right|<1$, which correspond to the energy levels localized near the impurity spins. We derived and analysed the conditions of the appearance of the energy states, localized near the impurity spins. The localized levels emerge at rather strong Ising interaction between chains. They appear below or above zone (6) at the critical values of model parameters $\alpha_{1}, \alpha_{2}, N$. For Eq. (5) these parameters are determined as the branches of two hyperbolas

$$
\left(\alpha_{1} \pm \frac{N}{N+1}\right)\left(\alpha_{2} \pm \frac{N}{N+1}\right)=\frac{1}{(N+1)^{2}} .
$$

\section{Transfer-matrix}

The special structure of the model Hamiltonian permits us to use standard transfer-matrix technique for numerical simulation of the model's thermodynamics. Similar to what was done in $[4,5]$ by means of a standard transfer-matrix scheme for decorated Heisenberg-Ising 1D spin models, we obtained the exact partition function of the systems considered

$$
\begin{aligned}
Z & =\operatorname{Tr} T^{L}, \quad T\left(\sigma_{l}, \sigma_{l+1}\right)=\exp \left(-\frac{E_{0}\left(\sigma_{l}, \sigma_{l+1}\right)}{T}\right) \\
& \times \prod_{\lambda}\left(1+\exp \left(-\frac{\varepsilon_{\lambda}\left(\sigma_{l}, \sigma_{l+1}\right)}{T}\right)\right) .
\end{aligned}
$$

Here $T\left(\sigma_{l}, \sigma_{l+1}\right)$ are transfer-matrix elements, which are in fact the partition functions of the finite XX chains with the Hamiltonian (3). To find, for example, free energy per spin for finite number $L$ of XX chains one needs all eigenvalues of transfer-matrix $T\left(\sigma_{l}, \sigma_{l+1}\right)$ :

$$
F=-\frac{T}{(N+1) L} \ln \left(\sum_{i=1}^{2 S+1} \lambda_{i}^{L}\right),
$$

According to our numerical calculations, the temperature dependences of the magnetization and the specific heat depend weakly on the number of unit cells $L$. For local characteristics we restricted our study to the infinite system and used only maximum eigenvalue of the transfer-matrix.

\section{Low-temperature thermodynamics}

Using above results, we performed numerical simulation of the field and the temperature dependences of the magnetization and the heat capacity for our system. One may expect the big effect of impurities when localized levels exist. For antiferromagnetic Ising interaction, the field dependence of the magnetization at very low temperatures demonstrates a jump associated with the spin-flip of impurity spins in sufficiently strong magnetic field. We presented field dependence of magnetization per spin for finite number of XX chains at low temperature in Fig. 1.

According to Fig. 1 dashed lines almost coincide with the corresponding solid lines at very low temperatures.

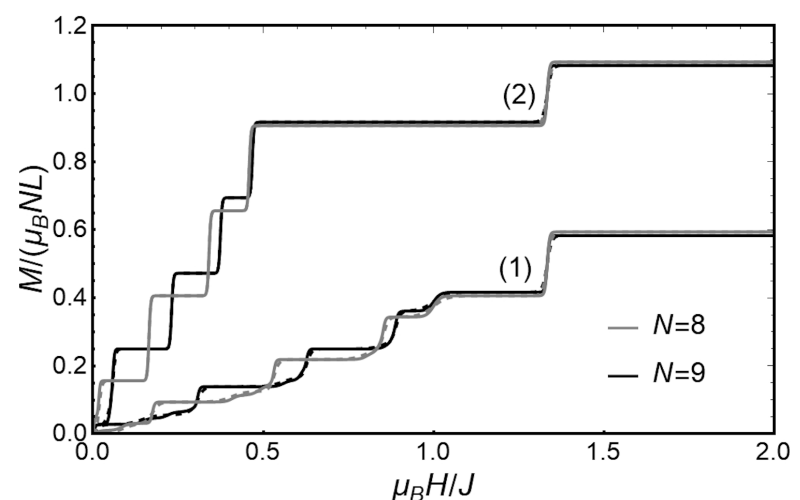

Fig. 1. Field dependence of magnetization per spin at $T / J=0.005$ for $S=1 / 2, L=3, J_{0} / J=-2, g_{0}=1.5$ for $g=1$ - curve $1, g=2$ - curve 2 , for $N=8$ - gray lines and for $N=9$ - black lines. Dashed lines correspond to one finite XX chain with effective impurities at the both ends (see [6]).

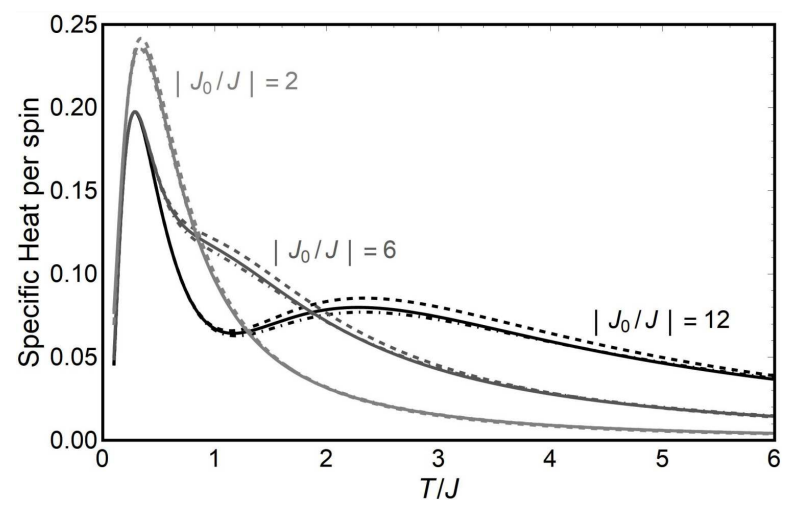

Fig. 2. Temperature dependence of specific heat per spin in dimensionless units at zero magnetic field, $S=$ $1 / 2, L=3, N=7$ for three different values of the Ising interactions (solid lines). Dashed lines indicate the specific heat for infinite number XX chain with the same number of spins and dashed-dotted lines correspond to $\mathrm{XX}$ chain with effective impurities at the both ends.

We found the possibility of two-peak behavior for zerofield temperature dependence of specific heat (see Fig. 2) at sufficiently strong antiferromagnetic Ising coupling.

We performed numerical simulation of the field dependence of thermodynamic average value $\left\langle\sigma_{l}^{z}\right\rangle$ for above model and found the possibility of oscillatory behavior of this quantity in the weak fields and rather small values of $J_{0}$ at low temperatures (see Figs. 3 and 4 ) for $S=1 / 2$ and $S=1$, respectively.

This effect can be explained by the competition of the antiferromagnetic interactions of these spins with the XX chain and applied weak external magnetic field. Note also that the above oscillatory behavior of local characteristic $\left\langle\sigma_{l}^{z}\right\rangle / S$ for all the impurity Ising spins is similar to the unstable behavior of this quantity for several 1D XX spin systems with one Ising impurity spin studied in $[6,7]$. 


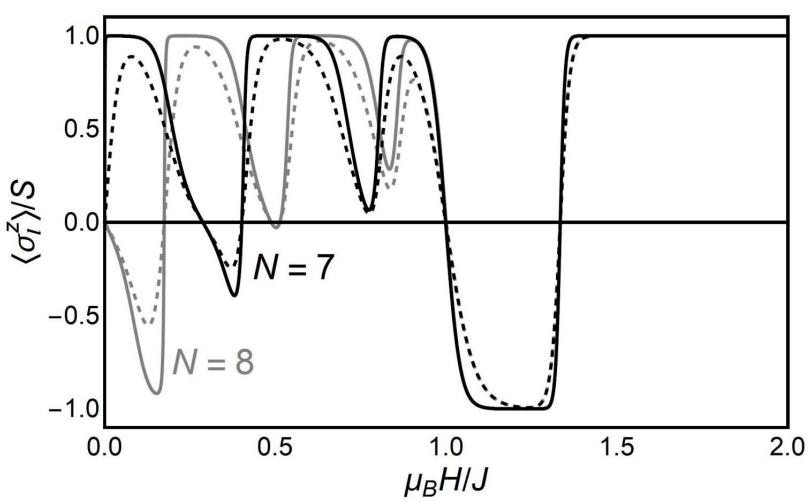

Fig. 3. Field dependence of $\left\langle\sigma_{l}^{z}\right\rangle / S$ for infinite system with $S=1 / 2$ at $T / J=0.01$ and $J_{0} / J=-2, g=1$, $g_{0}=1.5$ for two different sizes of XX chains $(N=7,8)$. Dashed lines indicate one XX chain with effective Ising impurities located at the both ends.

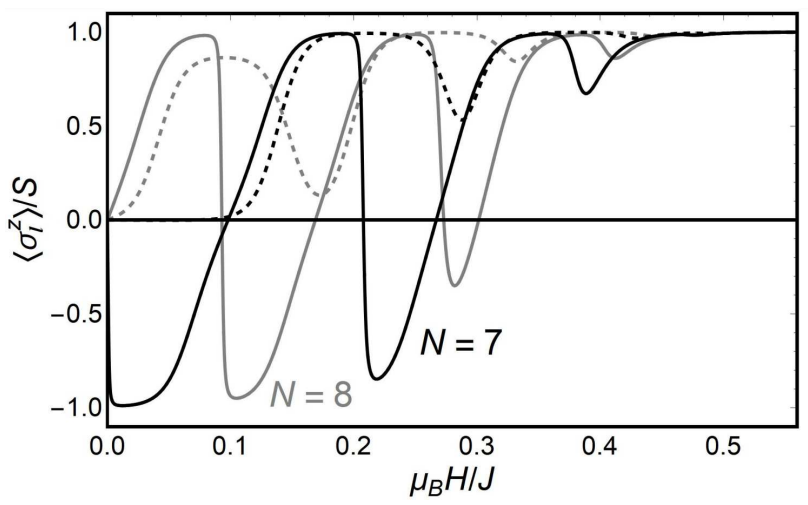

Fig. 4. Field dependence of $\left\langle\sigma_{l}^{z}\right\rangle / S$ for infinite system with $S=1$ at $T / J=0.01$ and $J_{0} / J=-0.2, g=2$, $g_{0}=0.5$ for two different sizes of XX chains $(N=7,8)$. Dashed lines indicate one XX chain with effective Ising impurities located at the both ends.

\section{Conclusions}

This work is devoted to the analytical and numerical study of 1D exactly solvable quantum spin model: a set of finite XX spin- $1 / 2$ chains connected by the Ising spins $S$ into the "ring". We derived the corresponding exact dispersion relation and determined the conditions of the appearance of the localized energy levels. We also performed numerical simulation for the low-temperature magnetic properties of the model proposed with the help of standard transfer matrix technique. Our analyses may be treated as a generalization of the analysis given in our previous works $[6,7]$.

\section{Acknowledgments}

The authors acknowledge the support of the VolkswagenStiftung, Germany (via grant 151110).

\section{References}

[1] A.A. Zvyagin, Quantum Theory of One-Dimensional Spin Systems, Cambridge Sci. Publ., Cambridge 2010.

[2] S.M. Giampaolo, F. Illuminati, New J. Phys. 12, 025019 (2010).

[3] C. Shi-Rong, X. Yun-Jie, M. Zhong-Xiao, Chin. Phys. B 19, 050304 (2010).

[4] J. Strečka, M. Jaščur, J. Magn. Magn. Mater. 272, 987 (2004).

[5] V.O. Cheranovskii, E.V. Ezerskaya, D.J. Klein, A.A. Kravchenko, J. Magn. Magn. Mater. 323, 1636 (2011).

[6] E.V. Ezerskaya, Acta Phys. Pol. A 131, 928 (2017).

[7] A.V. Artemov, E.V. Ezerskaya, Low. Temp. Phys. 44, 1645 (2018). 